\title{
Combination of microRNA-21 expression with the serum marker CA19-9 increases the accuracy of diagnosis of pancreatic adenocarcinoma
}

\author{
V.B. Filiputti ${ }^{1}$, M.L.A. Cirino ${ }^{1}$, F.S. Lizarte Neto ${ }^{1}$, P.C. Novais ${ }^{1,2}$, \\ L.P. Turra ${ }^{1}$, M.F.G.S. Tazima ${ }^{1}$, F.M. Peria ${ }^{1}$, D.P.C. Tirapelli ${ }^{1}$, \\ R. Kemp ${ }^{1}$, J.S. dos Santos ${ }^{1}$ and A.K. Sankarankutty ${ }^{1}$ \\ ${ }^{1}$ Departamento de Cirurgia e Anatomia, Faculdade de Medicina de Ribeirão \\ Preto, Universidade de São Paulo, Ribeirão Preto, SP, Brasil \\ ${ }^{2}$ Programa de Pós-Graduação em Interações Estruturais e Funcionais em \\ Reabilitação, Universidade de Marília, Marília, SP, Brasil
}

Corresponding author: A.K. Sankarankutty

E-mail: lab.biomol.cirurgia@fmrp.usp.br

Genet. Mol. Res. 19 (4): gmr18645

Received March 24, 2020

Accepted September 23, 2020

Published November 30, 2020

DOI http://dx.doi.org/10.4238/gmr18645

ABSTRACT. Pancreatic adenocarcinoma (PA) is one of the most aggressive malignant tumors of the digestive tract, with a five-year survival rate of $5 \%$. Early detection offers better possibilities of cure; however, in early stages it does not present symptoms, making the management of these patients difficult. The most commonly used biomarker for diagnosis of PA is serum CA19-9, which has a sensitivity between 70 and $80 \%$, although, with less than $50 \%$ specificity, due to its expression in various other types of cancer. The reduced rate of apoptosis plays a crucial role in carcinogenesis, in which the cytotoxic attack and the effectiveness of pharmacological treatment are reduced. Therefore, it would be useful to associate the biomarker CA19-9 with other molecular tools to increase the accuracy of the diagnosis, such as microRNAs (miRNAs) that target apoptotic genes. The association between the expression profile of anti-apoptotic microRNAs (miRNA-15a and miRNA-16), proapoptotic microRNAs (miRNA-21, miRNA-221 and miRNA-222) and serum CA19-9 was evaluated for the diagnosis of pancreatic ductal adenocarcinoma. Twenty pancreatic tumor tissue samples, 
after microdissection from patients diagnosed with pancreatic adenocarcinoma, were studied. As a control, seven samples of normal pancreas from cadaveric donors or patients undergoing pancreatic resection due to trauma were used. Serum CA19-9 was measured and the expression analysis of miRNA-21, miRNA-221, miRNA-222, miRNA-15a and miRNA-16 was measured using real-time quantitative PCR. The usefulness of pro-apoptotic and anti-apototic microRNAs alone or in association with CA19-9 in the diagnosis of PA was evaluated. The expression of miRNA-21 was significantly associated with PA, when compared with control samples. There was no significant difference in the expression of the other miRNAs. We conclude that association of CA19-9 and microRNA-21expression would increase sensitivity and accuracy in the diagnosis of PA.

Key words: Pancreatic ductal adenocarcinoma; miRNAs; Apoptosis; CA 19-9

\section{INTRODUCTION}

Pancreatic adenocarcinoma (PA) is ranked as the 14th most common cancer and the 7 th leading cause of cancer mortality in the world, and is expected to become the second leading cause of death for cancer patients by 2030 (Mcguigan et al., 2018, Li et al., 2019).

Overall five-year survival is less than $5 \%$, but in patients with small tumors with negative margin resection and tumor-free ganglia, survival can reach 15-20\% (Brand et al., 2011; Jamieson et al., 2011; Ryu et al., 2011; Papaconstantinou et al., 2013). The poor prognosis of patients with PA is mainly attributed to identification and diagnosis at an advanced stage, in combination with the invasive and aggressive nature of the neoplasia coupled with the resistance of pancreatic cancer cells to chemotherapy and radiation therapies, with $60 \%$ of patients with advanced stage of the disease having a survival rate between 3 and 6 months (Du Rieu et al., 2010; Rawat et al., 2019). Early detection is the best option to cure PA; however, in its early stages, this cancer is asymptomatic. Progress has been made towards detection of precursor lesions, represented by mucinous papillary intraductal neoplasia, cystic mucinous neoplasia, and pancreatic intraductal neoplasia; the latter is a noninvasive microscopic epithelial neoplasm located in the small pancreatic ducts with atypical cytology and morphology (Du Rieu et al., 2010). The importance of early detection is reiterated by the fact that its genetic evolution and metastasis appear relatively late, thus generating a window of opportunity for therapeutic intervention with healing potential (Matthaei et al.,, 2011). Even with the use of refined diagnostic devices, early diagnosis of pancreatic adenocarcinoma remains rare, being that 80 to $90 \%$ of patients at the time of diagnosis have a locally advanced tumor. Thus, only $10 \%$ of patients can undergo the surgical procedure, and unfortunately only $15-20 \%$ of patients undergoing resection remain tumor free after 5 years (Ouaïssi et al., 2012). The most commonly used biomarker for diagnosis is serum CA19-9. This marker has a sensitivity for pancreatic cancer between 70 and $80 \%$, but with a specificity of less than $50 \%$, since when correlated with the size and staging of the tumor, it becomes ineffective for the early stages of the disease (Liu et al., 2012). 
The increase in the expression of this marker associated with nonmalignant conditions, such as pancreatitis and obstructive jaundice, and its inability to detect tumors in early stages, are its limitations. In addition, its use is also ineffective in patients with Lewis negative genotype, with tumors that do not express this antigen, representing $5-10 \%$ of cases (Brand et al., 2011).

It is believed that the only alternative to cure is surgical treatment; however, only $10 \%$ of patients undergo this procedure, where the five-year survival rate remains between $7 \%$ and $34 \%$, compared with the survival of patients of 3-11 months for unresected cancer (Ouaïssi et al., 2012). The high rate of local recurrence and metastasis are causes for poor prognosis. Neoadjuvant chemoradiotherapy has been used as a preoperative alternative for tumor volume reduction, micro-metastasis treatment and increased likelihood of complete resection (Zhao et al., 2012).

It is currently believed that induction of apoptosis in tumor cells is a key to a new form of treatment, as this type of programmed cell death is a fundamental process in maintaining tissue homeostasis (Yan et al., 2009; Vaccaro et al., 2012).

The mechanisms of avoidance of apoptosis involve apoptotic disorders, mainly the reduction of caspases and signaling to the impaired death receptor, in other words, resistance to apoptosis is one of the pillars that justify the invasiveness of pancreatic cancer (Słotwiński and Słotwińska, 2016)

Hypoexpression of the RASSF6 gene in the PA is associated with low survival, suggesting that the RASSF6 is a prognostic indicator in patients with PA undergoing radical surgeries. The RASSF6 gene, downregulated by $30-60 \%$ in several solid tumors, also acts as an apoptosis regulator in the NF- $\mathrm{KB}$ pathway during the fibroinflammatory process. Thus, the intracellular signaling pathways that delay apoptosis contribute to the elucidation of tumorigenesis (Słotwiński and Słotwińska, 2016).

Pancreatic adenocarcinoma is a set of genetic and epigenetic alterations, as well as the miRNAs, which are totally linked to cell proliferation, differentiation, and apoptosis. Its importance in cancer is due to the fact that $50 \%$ of miRNA genes are located in fragile regions associated with the disease (Tavano et al., 2012).

The importance of miRNAs is related to the location of their genes in cancerassociated sites, and generally altered genomic regions, where deletions, local amplifications, and chromosome breakdown in certain microRNA-coding regions, suggest a direction for the study of miRNAs in tumorigenesis (Aqeilan et al., 2010; Bhat et al., 2012). In this context, the reduced rate of apoptosis plays a crucial role in carcinogenesis, and is one of the most important features acquired by pancreatic cancer cells, which protects these cells from attack by the immune system and reduces the effectiveness of pharmacological treatment. Therefore, it is necessary to associate the CA19-9 biomarker with molecular tools that increase diagnostic accuracy, such as microRNAs that target apoptotic genes. We analyzed the association of expression profiles of anti-apoptotic microRNAs (miRNA-15a, miRNA-16), pro-apoptotic microRNAs (miRNA-21, miRNA221 and miRNA-222) and the CA19-9 biomarker for the diagnosis of pancreatic ductal adenocarcinoma.

\section{MATERIAL AND METHODS}

\section{Patients}


From 2008 to 2012, tumor tissue samples from 20 patients with pancreatic adenocarcinoma were collected for the study. The control group included normal pancreatic tissue from seven patients, either organ donors or trauma victims. After confirming the histopathological diagnosis, the samples were microdissected and separated for later RNA extraction and analysis of miRNA-15a, miRNA-16, miRNA-211, miRNA-221 and miRNA222 expression. The project was approved by the Research Ethics Committee of the Clinical Hospital of Ribeirão Preto (process no. 8054/2010) and the participants of this research consented to donate the tumor fragments by signing the Free and Informed Consent Term, before the tumor resection surgery.

\section{RNA extraction in tissue}

To the samples were added $250 \mu \mathrm{L}$ PBS (Saline Phosphate Buffer) and $750 \mu \mathrm{L}$ Trizol® (Invitrogen, USA). To the homogenized fragment, $200 \mu \mathrm{L}$ chloroform was added, in which the (supernatant) aqueous phase of each vial was transferred to new tubes. RNA was precipitated with $500 \mu \mathrm{L}$ of $100 \%$ isopropyl alcohol and remained (at $-20^{\circ} \mathrm{C}$ ) for at least $12 \mathrm{~h}$. The next day, the sample was centrifuged at $4^{\circ} \mathrm{C}$ for $15 \mathrm{~min}$ at $14,000 \mathrm{rpm}$, discarding the supernatant. Then we added $1000 \mu \mathrm{L}$ of $75 \%$ ethanol followed again by refrigerated centrifugation at $4^{\circ} \mathrm{C}$. The upper phase was discarded and the dried precipitate dissolved with DEPC (dimethylpyrocarbonate) treated water for at least $15 \mathrm{~min}$. To verify the integrity of the obtained RNA, each sample was submitted to $1 \%$ agarose gel electrophoresis at the end of the step described above. We also used a device called Thermo Scientific Nano Drop 2000, a spectrophotometer that provides the RNA concentration in a 1 to $2 \mu \mathrm{L}$ sample.

\section{Complementary DNA (cDNA) synthesis of microRNAs: miRNA-21, miRNA- 221, miRNA-222, miRNA-15a and miRNA-16}

For synthesis of microRNA cDNA (complementary DNA), reverse transcription was performed using the commercial High Capacity cDNA Reverse Transcription kit (Applied Biosystems).

\section{MicroRNA RQ: miRNA-21, miRNA-221, miRNA-222, miRNA-15a and miRNA-16}

From the cDNA obtained from the samples, quantitative real-time polymerase chain reaction (PCR) amplification (RQ-PCR) was performed using the TaqMan Master Mix reagent (Applied Biosystems). For the quantitative expression analysis, commercially available TaqMan Assay-on-demand systems composed of oligonucleotides and probes (Applied Biosystems) were used.

\section{Statistical analysis}

Statistical analysis of gene expression by the quantitative real-time PCR method was performed by the One-way ANOVA method, Mann-Whitney Test using GraphPad Prism version 8.00 for Windows, (GraphPad Software, San Diego California USA). Values 
of $\mathrm{P}<0.05$ were considered statistically significant. Sensitivity, specificity, positive predictive value, negative predictive value, and Receiver Operating Characteristic (ROC) curve were calculated to assess the diagnostic performance of CA19-9 and the evaluated microRNAs.

\section{RESULTS}

Regarding the expression of microRNAs, significant hyperexpression $(\mathrm{P}=0.0161$, Mann Whitney test) was detected in the expression of miRNA-21 between the patients diagnosed with pancreatic adenocarcinoma and the control patients (Figure 1).

The ROC curve for variable CA19-9, where AUC is $=0.904(\mathrm{P}=0.002)$, with $70 \%$ sensitivity and $100 \%$ specificity for values above 37 (Figure 2), while for miRNA21 variable, where AUC is $=0.82(\mathrm{P}=0.013)$, with sensitivity $65 \%$ and specificity $86 \%$ for values above 1.35 (Figure 3 ).
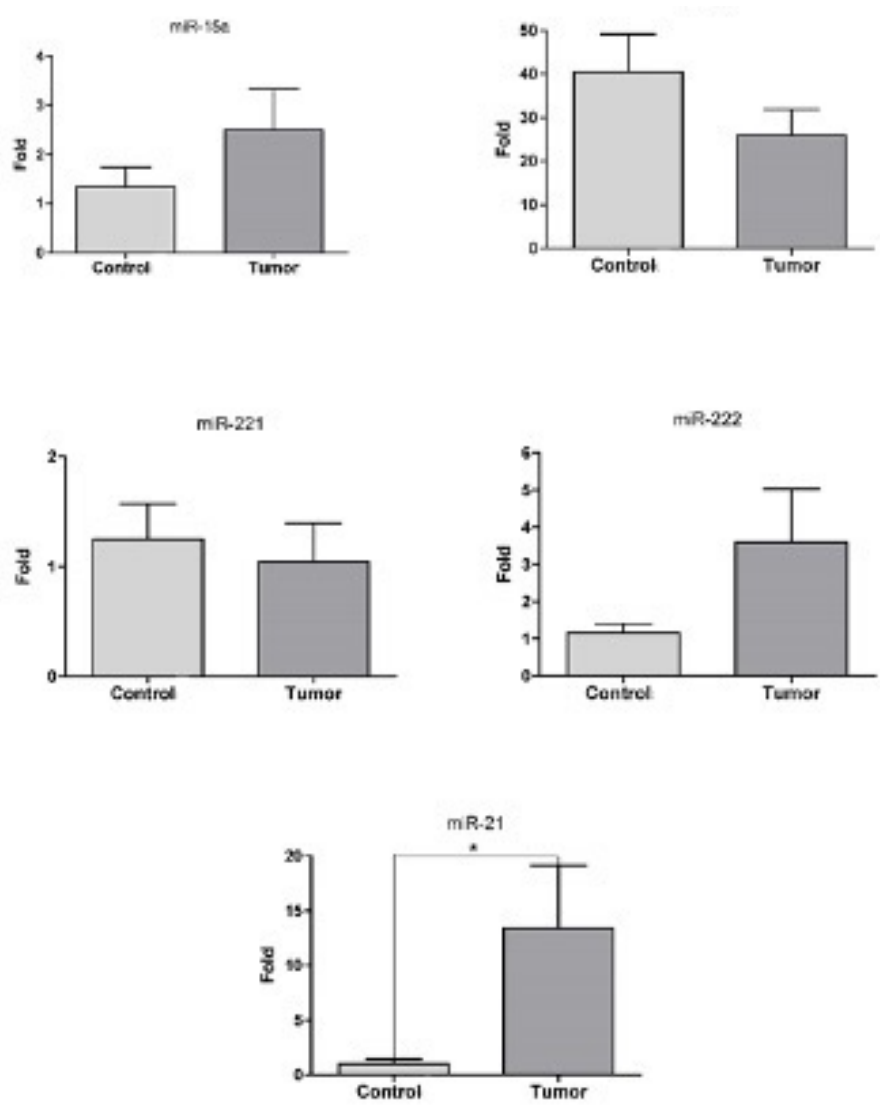

Figure 1. Representation of mean values ( \pm standard deviation) of tissue expression of microRNAs: miRNA-15a, miRNA-16, miRNA-221, miRNA-222 and miRNA-21 among the groups studied. There was a significant difference $(p=0.0161)$ in miRNA-21 expression between the group of patients diagnosed with pancreatic adenocarcinoma when compared with the control group $(*)$. 


\section{ROC Curve}

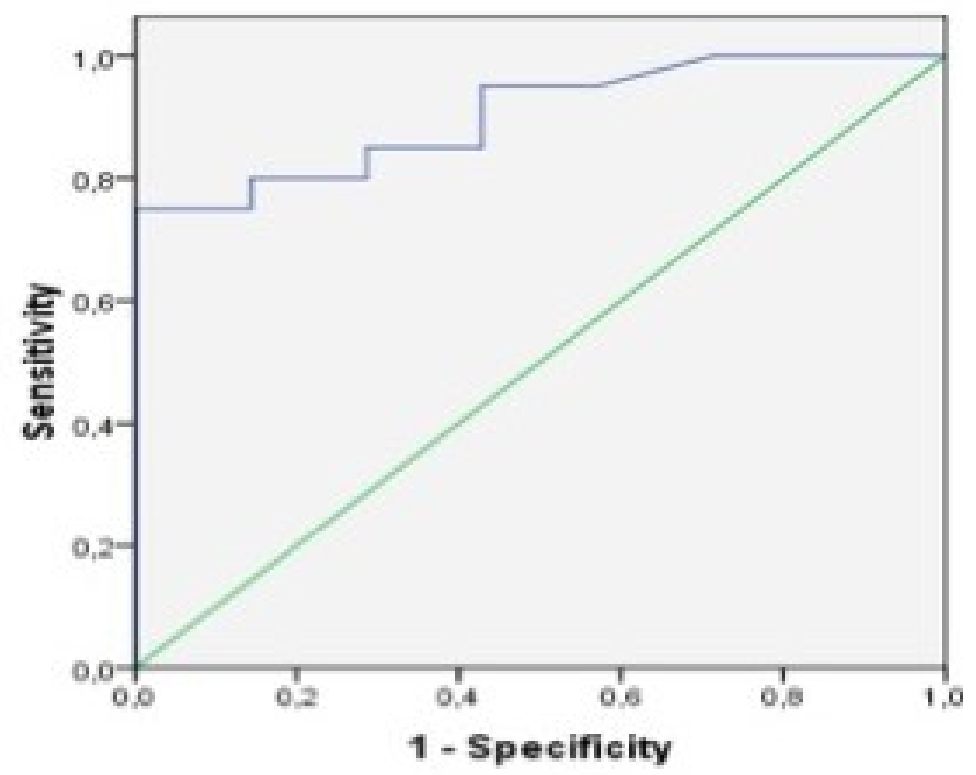

Figure 2. ROC curve - CA19-9: Tumor vs. Control

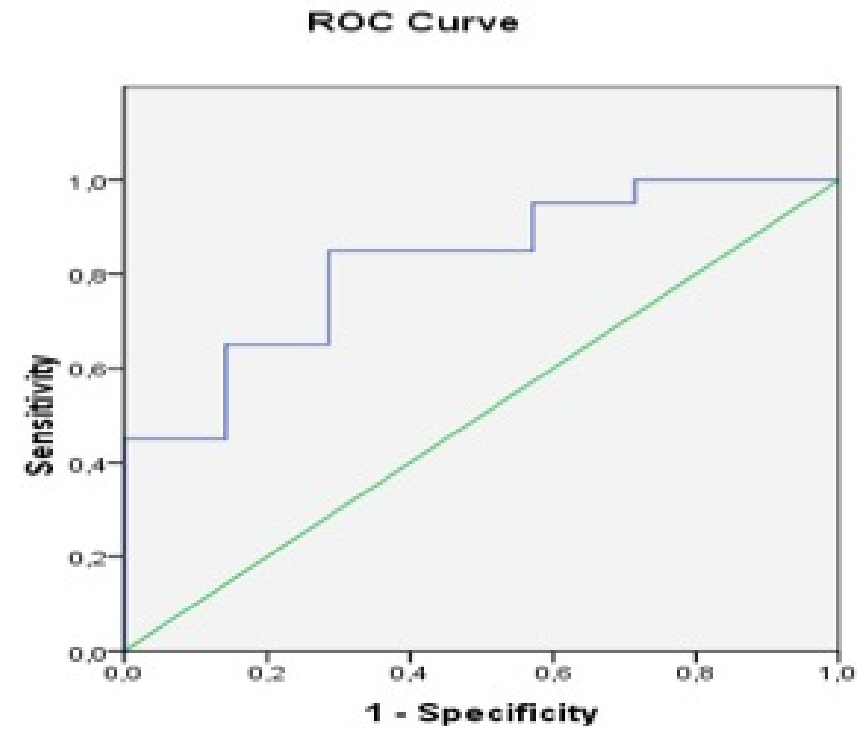

Figure 3. ROC curve - miRNA-21: Tumor vs. Control 
The ROC curve for variable miRNA15a, where AUC is $=0.529(\mathrm{P}=0.825)$ (Figure 4), for variable miRNA16, where $\mathrm{AUC}$ is $=0.693(\mathrm{P}=0.135)$ (Figure 5), for variable miRNA221, where AUC is $=0.686(\mathrm{P}=0.15)$ (Figure 6) and for the miRNA222 variable, where AUC is $=0.543(\mathrm{P}=0.74)$ (Figure 7), it can demonstrate that these markers do not show capacity to discriminate between tumor and normal tissue. In this sample of patients, between the CA19-9 tumor marker and the miRNAs evaluated, only CA19-9 and miRNA21 were able to discriminate the tumor from normal pancreatic tissue. When evaluated alone, CA19-9 is better than miRNA-21, with a sensitivity ( 0.69 vs 0.70$)$, specificity ( 1 vs $0.86)$, positive predictive value ( 1 vs 0.94$)$, negative predictive value ( 1 vs 0.64$)$ and accuracy. (77\% vs $73 \%$ ). However, when both were evaluated together, the diagnostic performance is superior than each one alone; sensitivity (0.83), specificity (1), positive predictive value (1), negative predictive value (1) and accuracy (87\%) (Table 1).

Table 1. Serum expression of CA19-9 and miRNA-21 isolated and associated to determine the cutoff point according to the diagnostic accuracy of pancreatic cancer.

\begin{tabular}{|c|c|c|c|c|c|c|c|}
\hline & Assay & Cohort & Sensibility & Specificity & VPP & VPN & Accuracy \\
\hline Tumor & CA19-9 & 37 & $\begin{array}{l}0.69 \\
(0.47 ; 0.86)\end{array}$ & $\begin{array}{l}1.00 \\
(0.56 ; 1.00)\end{array}$ & $\begin{array}{l}1.00 \\
(1.00 ; 1.00)\end{array}$ & $1.00(0.74 ; 1.00)$ & $77 \%$ \\
\hline $\mathrm{X}$ & miRNA21 & 1.35 & $\begin{array}{l}0.70 \\
(0.47 ; 0.86)\end{array}$ & $\begin{array}{l}0.86 \\
(0.42 ; 1.00)\end{array}$ & $\begin{array}{l}0.94 \\
(0.87 ; 1.00)\end{array}$ & $\begin{array}{l}0.64 \\
(0.39 ; 0.89)\end{array}$ & $73 \%$ \\
\hline Control & CA19-9+miRNA21 & & $\begin{array}{l}0.83 \\
(0.60 ; 0.94)\end{array}$ & $\begin{array}{l}1.00 \\
(0.56 ; 1.00) \\
\end{array}$ & $\begin{array}{l}1.00 \\
(1.00 ; 1.00)\end{array}$ & $1.00(0.74 ; 1.00)$ & $87 \%$ \\
\hline
\end{tabular}

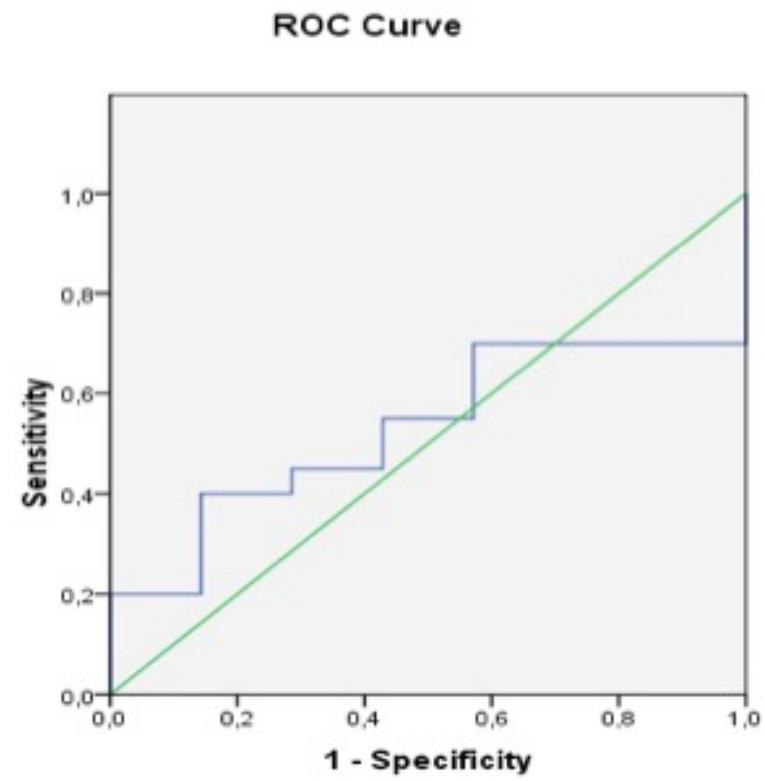

Figure 4. ROC curve - miRNA-15a: Tumor vs. Control 


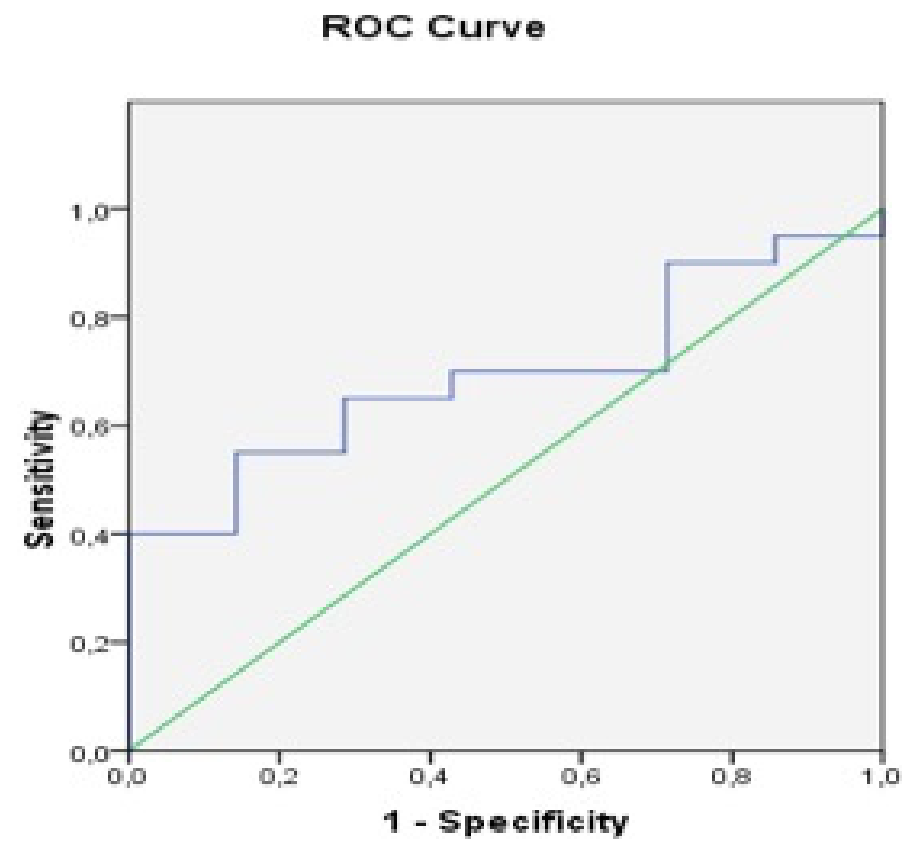

Figure 5. ROC curve - miRNA-16: Tumor vs. Control

ROC Curve

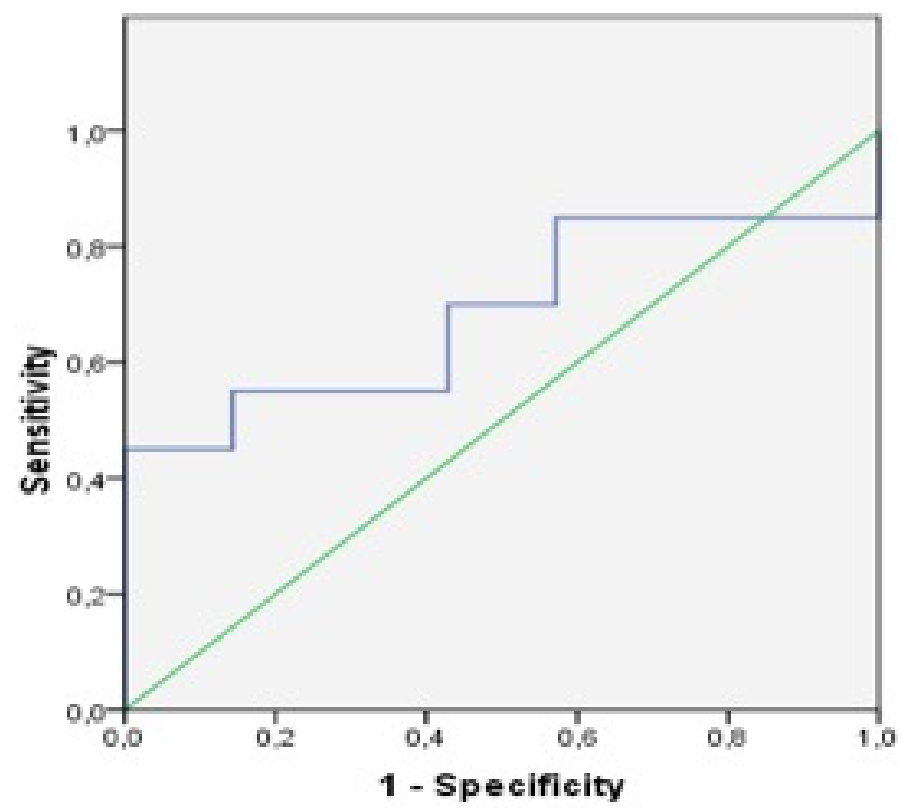

Figure 6. ROC curve - miRNA-221: Tumor vs. Control 


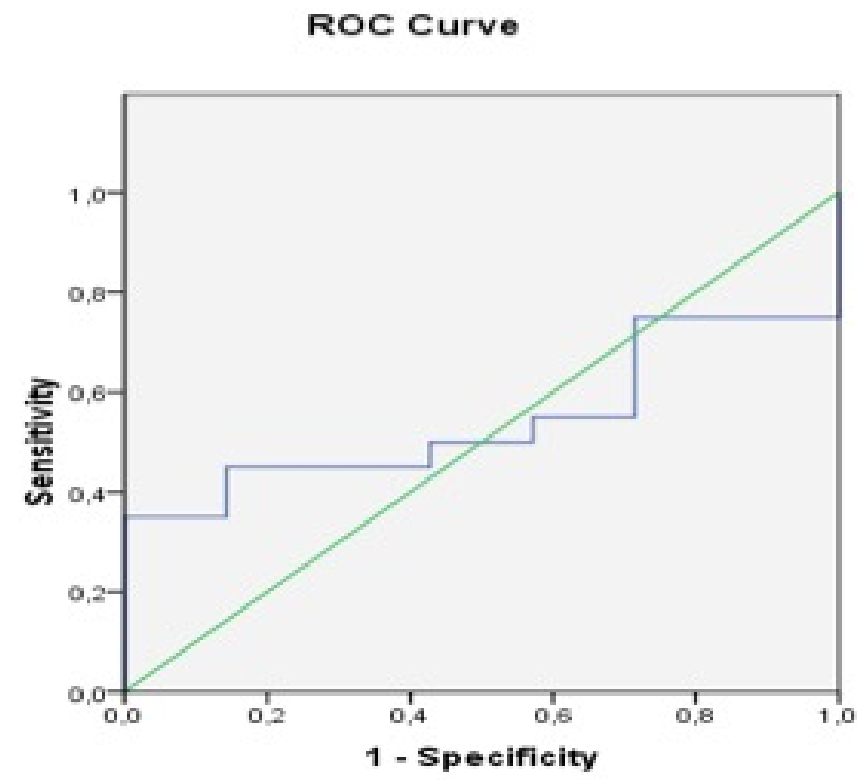

Figure 7. ROC curve - miRNA-222: Tumor vs. Control

\section{DISCUSSION}

The early stages of tumorigenesis are asymptomatic, hindering a possible early diagnosis that would give hope for successful therapy. Surgery performed in the early stages of the disease can significantly increase survival; however, at the time of diagnosis, the tumor is unresectable in $80 \%$ of patients and distant metastases already exist. The survival time of patients diagnosed with unresectable pancreatic tumor is on average six months and may only slightly increase after the application of complex chemotherapy. These data emphasize the need to pay more attention to the intracellular signaling pathways delaying apoptosis of cancer cells (Słotwiński and Słotwińska, 2016; Strobel et al., 2019).

Recent studies have sought to elucidate the role of the apoptosis mechanism in pancreatic adenocarcinoma by characterizing the expression profile of the microRNAs associated with this mechanism. In our results, we observed an increase in microRNA-21 expression in the group of patients diagnosed with PA when compared to the control group. Regarding microRNAs miRNA-221 and miRNA-222, even without statistical evidence, hyperexpression of miRNA-221 was noted in the control group and hyperexpression of miRNA-222 in the group of patients diagnosed with PA, our findings do not coincide with the reports in the literature, justified by phenotypic divergences between populations. In our study, pancreatic tissue samples from normal individuals were used as a control group, while other researchers used samples of paratumor tissues, which reduces the specificity of the marker, since the inflammatory process that circumscribes tumor cells can express the target in question with levels equivalent to the tumorigenic microenvironment, highlighting it in benign inflammatory conditions. Another fact that stands out in the control group, as it directly interferes with expression levels, since it is used, in many cases, as a calibrator in real-time PCR reactions (Bauer et al., 2018). miRNA-21 was analyzed in invasive 
pancreatic tissue and Vater papilla adenocarcinomas, comparing them to normal adjacent tissues. In both tumor tissues, there was serum hyperexpression of miRNA-21, correlating it with preoperative CA 19-9 levels, which suggests that miRNA-21 dysregulation may be associated with both histological characteristics and biological behavior of different pancreatic tumors (Tavano et al., 2012).

According to data from the Gene Expression Omnibus database, miRNA-21 has been reported as one of the top 20 microRNAs differentially expressed, revealed to regulate a range of biological behaviors in cancer cells, including proliferation, migration, invasion, metastasis and apoptosis. The new therapeutic approach to target microRNAs by antisensebased gene silencing has demonstrated that miRNA-21 induces cancer cells to apoptosis through activation of the caspase-8 apoptotic pathway (Ge et al., 2019). There are significant findings of hyperexpression of microRNAs miRNA-21, miRNA-221 and miRNA-222 in neoplastic tissues in relation to normal pancreas, in addition, high levels of miRNA-21 were related to stage T3 (Papaconstantinou et al., 2012). In cells of the side population (SP), the microRNAs miRNA-21 and miRNA-221 had highly significant expression, therefore, when transfecting antagomir-21 and antagomir-221 antagonists, it significantly reduced the differentiation of SP cells and thus reduced the proliferation and tumor invasion (Zhao et al., 2015). Overexpression of miRNA-221 was observed both in pancreatic neoplastic tissues and in commercial pancreatic cell lines (PK-45H, PANC-1, PK-59 KP4-1, PK-1, and NOR-P1), the same was true in the plasma of patients with PA compared to control (Kawaguchi et al., 2013).

Li et al., 2018, analyzed the expression of miRNA-221-3p in pancreatic cancer cell lines by comparing it with the expression of normal pancreatic ductal epithelial cells. miRNA $-221-3 p$ is upregulated in all analyzed pancreatic cell lines, resulting in increased cell proliferation and inhibition of apoptosis, while inhibition of miRNA-221-3p decreased the rate of cell growth and promoted apoptosis. The expression level of miRNA -221-3p was investigated in plasma and statistical analysis showed that miRNA-221-3p expression was correlated with distant metastatic and TNM phases. The ROC curves and the area under the ROC curve suggested that the diagnostic efficacy for distant metastases using miRNA 221-3p is better than CA19-9 (AUC: 0.689 vs. 0.587). In summary, it was discovered that miRNA -221-3p could serve as a biomarker for PA (Li et al., 2018).

Other research also investigated miRNA-221 as a potential biomarker for the detection of gastric cancer, with a sensitivity of $82.4 \%$ and specificity of $58.8 \%$. This data suggests that the serum miRNA-221 presents itself as a possible non-invasive biomarker for the early detection of gastric cancer and possibly other tumors of the digestive tract (Song et al., 2012).

As an early diagnostic marker, the authors stipulated the combination of microRNAs miRNA-16, miRNA-196a and serum CA19-9, plus the deregulation of microRNAs miRNA-21, miRNA-155 and miRNA-196 in lesions of IPMN (intraductal mucinous neoplasms) ) and PanIN (pancreatic intraepithelial neoplasia), suggesting using them as early non-invasive molecular tools for the diagnosis of pancreatic carcinoma (Słotwiński and Słotwińska, 2016).

In conclusion to the literature reviews, microRNAs as plasma biomarkers associated with CA 19-9 are able to distinguish neoplastic lesions from chronic pancreatitis, especially in the initial tumor screening. As evidenced by the combination of microRNAs miRNA-16, 
miRNA-196a and CA 19-9 (AUC $=0.956$; sensitivity, 88.4\%; specificity, 96.3\%) (Liu et al., 2012).

It is important to consider some limitations of the present study, since it is a case report and the number of samples is relatively small, in which the hypotheses raised need to be confirmed by larger studies and in other centers involving different phenotypic populations. Reliable diagnoses are directly related to positive and negative predictive values, although the prevalence of the disease in the population studied is not affected by sensitivity and specificity. Therefore, future studies need to include samples of benign inflammatory masses to assess the performance of biological markers in diagnostic discrimination. It is also necessary to invest in studies that address other microRNAs associated with the apoptosis mechanism to clarify their involvement in the development and progression of pancreatic cancer. As well as exploring the potential of anti-apoptotic and pro-apoptotic microRNAs in the diagnosis and treatment of pancreatic cancer.

The association of carcinoembryonic antigen (CEA), carbohydrate antigen 125 (CA 125) and CA19-9 maximizes the pre-surgical and chemotherapeutic prognostic results, however, this study and among others emphasize the importance of defining a set of biomarkers that include gene and epigenetic markers, since imaging tests become nonspecific in view of the accuracy of liquid biopsies, so that survival can be optimized based on early diagnosis of PA (Chang et al., 2017).

\section{CONCLUSIONS}

The group of patients diagnosed with PA had higher serum levels of CA19-9 and higher microRNA-21 expression when compared to the control group. The combination of CA19-9 and microRNA-21 increases sensitivity and accuracy in the diagnosis of PA. No differences were detected between the groups studied in microRNA expression: miRNA15a, miRNA-16, miRNA-221 and miRNA-222.

\section{CONSENT}

All the patients allowed personal data processing, and an informed consent form was obtained from all individual participants included in the study.

\section{FUNDING STATEMENT}

Our research did not receive any specific funding.

\section{CONFLICTS OF INTEREST}

The authors declare no conflict of interest.

\section{REFERENCES}

\footnotetext{
Aqeilan RI, Calin GA and Croce CM (2010). miR-15a and miR-16-1 in cancer: discovery, function and future perspectives. Cell Death Differ. 17: 215-220.

Bauer AS, Nazarov PV, Giese NA, Beghelli S, et al. (2018). Transcriptional variations in the wider peritumoral tissue environment of pancreatic cancer. Int. J. Cancer. 142: 1010-1021.
} 
Bhat K, Wang F, Ma Q, Li Q, et al (2012). Advances in biomarker research for pancreatic cancer. Curr. Pharm. Des. 18: 2439-2451.

Brand RE, Nolen BM, Zeh HJ, Allen PJ, et al. (2011). Serum biomarker panels for the detection of pancreatic cancer. Clin. Cancer Res. 17: 805-816.

Chang JC and Kudranda M (2017). Novel diagnostic and predictive biomarkers in pancreatic adenocarcinoma. Int. J. Mol. Sci. 18: 667.

Du Rieu MC, Torrisani J, Selves J, Al Saati T, et al. (2010). MicroRNA-21 is induced early in pancreatic ductal adenocarcinoma precursor lesions. Clin. Chem. 56: 603-612.

Ge JH, Zhu JW, Fu HY, Shi WB, et al. (2019). An Antisense Oligonucleotide Drug Targeting miR-21 Induces H1650 Apoptosis and Caspase Activation. Technol Cancer Res. Treat. 18: 1533033819892263.

Jamieson NB, Carter CR, Mckay CJ and Oien KA (2011) Tissue biomarkers for prognosis in pancreatic ductal adenocarcinoma: a systematic review and meta-analysis. Clin. Cancer Res. 17: 3316-3331.

Kawaguchi T, Komatsu S, Ichikawa D, Morimura R, et al. (2013). Clinical impact of circulating miR-221 in plasma of patients with pancreatic cancer. Br. J. Cancer. 108: 361-369.

Li F, Xu JW, Wang L, Liu H, et al. (2018). MicroRNA-221-3p is up-regulated and serves as a potential biomarker in pancreatic cancer. Artif Cells Nanomed Biotechnol. 46: 482-487.

Li JT, Wang YP, Yin M and Lei QY (2019). Metabolism remodeling in pancreatic ductal adenocarcinoma. Cell Stress. 3: $361-368$

Liu J, Gao J, Du Y, Li Z, et al. (2012). Combination of plasma microRNAs with serum CA19-9 for early detection of pancreatic cancer. Int. J. Cancer. 131: 683-691.

Matthaei H, Schulick RD, Hruban RH and Maitra A (2011). Cystic precursors to invasive pancreatic cancer. Nat. Rev. Gastroenterol Hepatol. 8: 141-150.

Mcguigan A, Kelly P, Turkington RC, Jones C, et al. (2018). Pancreatic cancer: A review of clinical diagnosis, epidemiology, treatment and outcomes. World J. Gastroenterol. 24: 4846-4861.

Ouaïssi M, Giger U, Louis G, Sielezneff I, et al. (2012). Ductal adenocarcinoma of the pancreatic head: a focus on current diagnostic and surgical concepts. World J. Gastroenterol. 18: 3058-3069.

Papaconstantinou IG, Manta A, Gazouli M, Lyberopoulou A, et al. (2013). Expression of microRNAs in patients with pancreatic cancer and its prognostic significance. Pancreas. 42: 67-71.

Rawat M, Kadian K, Gupta Y, Kumar A, et al. (2019). MicroRNA in Pancreatic Cancer: From Biology to Therapeutic Potential. Genes. 10: 752.

Ryu JK, Matthaei H, Dal Molin M, Hong SM, et al. ( 2011). Elevated microRNA miR-21 levels in pancreatic cyst fluid are predictive of mucinous precursor lesions of ductal adenocarcinoma. Pancreatology. 11:343-350.

Song MY, Pan KF, Su HJ, Zhang L, et al. (2012). Identification of serum microRNAs as novel non-invasive biomarkers for early detection of gastric cancer. PLoS One. 7: e33608.

Słotwiński R and Słotwińska SM (2016). Diagnostic value of selected markers and apoptotic pathways for pancreatic cancer. Cent. Eur. J. Immunol. 41: 392-403, 2016.

Strobel O, Neoptolemos J, Jäger D and Büchler MW (2019). Optimizing the outcomes of pancreatic cancer surgery. Nat. Rev. Clin. Oncol. 16(1): 11-26.

Tavano F, Di Mola FF, Piepoli A, Panza A, et al. (2012). Changes in miR-143 and miR-21 expression and clinicopathological correlations in pancreatic cancers. Pancreas. 41: 1280-1284.

Vaccaro V, Gelibter A, Bria E, Iapicca P, et al. (2012). Molecular and genetic bases of pancreatic cancer. Curr. Drug Targets. 13: 731-743.

Yan LH, Li L, Xie YB, Xiao Q, et al. (2009). [Effects of E2F-1 overexpression on apoptosis of gastric cancer cells and expressions of apoptosis-related genes]. Ai Zheng. 28: 1176-1180.

Zhao Q, Rashid A, Gong Y, Katz MH, et al. (2012). Pathologic complete response to neoadjuvant therapy in patients with pancreatic ductal adenocarcinoma is associated with a better prognosis. Ann Diagn. Pathol. 16: 29-37.

Zhao Y, Zhao L., Ischenko I, Bao Q, et al. (2015). Antisense inhibition of microRNA-21 and microRNA-221 in tumorinitiating stem-like cells modulates tumorigenesis, metastasis, and chemotherapy resistance in pancreatic cancer. Target Oncol. 10: 535-548. 